\title{
Emergent 1/3 magnetization plateaus in pyroxene $\mathrm{CoGeO}_{3}$
}

\author{
H. Guo $\odot,{ }^{1,2}$ L. Zhao, ${ }^{1}$ M. Baenitz, ${ }^{1}$ X. Fabrèges,${ }^{3}$ A. Gukasov $\odot,{ }^{3}$ A. Melendez Sans $\odot,{ }^{1}$ \\ D. I. Khomskii $\odot{ }^{4}{ }^{4}$ L. H. Tjeng $₫{ }^{1}{ }^{1}$ and A. C. Komarek ${ }^{1}{ }^{1, *}$ \\ ${ }^{1}$ Max-Planck-Institute for Chemical Physics of Solids, Nöthnitzer Strasse 40, D-01187 Dresden, Germany \\ ${ }^{2}$ Neutron Science Platform, Songshan Lake Materials Laboratory, Dongguan, Guangdong 523808, China \\ ${ }^{3}$ Laboratoire Léon Brillouin, CEA-CNRS, CE-Saclay, F-91191 Gif-sur-Yvette, France \\ ${ }^{4}$ Physics Institute II, University of Cologne, Zülpicher Strasse 77, D-50937 Cologne, Germany
}

(Received 2 April 2021; revised 28 June 2021; accepted 19 July 2021; published 11 August 2021)

\begin{abstract}
Despite the absence of an apparent triangular pattern in the crystal structure, we observe unusually wellpronounced 1/3 magnetization plateaus in the quasi-one-dimensional Ising spin chain compound $\mathrm{CoGeO}_{3}$ which belongs to the class of pyroxene minerals. We succeeded in uncovering the detailed microscopic spin structure of the $1 / 3$ magnetization plateau phase by means of neutron diffraction. We observed changes of the initial antiferromagnetic zero-field spin structure that resemble a regular formation of antiferromagnetic "domain wall boundaries," resulting in a kind of modulated magnetic structure with a $1 / 3$-integer propagation vector. The net ferromagnetic moment emerges at these "domain walls" whereas two thirds of all antiferromagnetic chain alignments can be still preserved. We propose a microscopic model on the basis of an anisotropic frustrated square lattice to explain the observations.
\end{abstract}

DOI: 10.1103/PhysRevResearch.3.L032037

Magnetization plateaus at finite magnetic field have attracted enormous attention, both theoretically and experimentally [1]. The phenomenon of magnetization jumps to rational values $(1 / n)$ of the saturation magnetization $\left(M_{S}\right)$ during a magnetization process is intimately connected to the presence of frustration in quantum magnets. Here, one may distinguish between systems with "geometric frustration" as a result of the crystal structure, e.g., triangular lattices, and those with "interaction frustration," where the presence of several exchange interactions lead to a competition for the ground state [2]. Examples for the first category include $\mathrm{Dy}_{2} \mathrm{Ti}_{2} \mathrm{O}_{7}$ [3], $\mathrm{CdCr}_{2} \mathrm{O}_{4}$ [4], $\mathrm{Ba}_{3} \mathrm{CoSb}_{2} \mathrm{O}_{9}$ [5], and $\mathrm{Ca}_{3} \mathrm{Co}_{2} \mathrm{O}_{6}$ [6-15], and for the second category $\mathrm{SrCu}_{2}\left(\mathrm{BO}_{3}\right)_{2}$ [16-19] and $\mathrm{TbB}_{4}$ $[20,21]$. Other examples are listed in Refs. [1,2].

Here, we studied the $S=3 / 2$ system $\mathrm{CoGeO}_{3}$ which belongs to the family of pyroxene minerals [22]. Pyroxenes $A M X_{2} \mathrm{O}_{6}$ ( $A=$ mono- or divalent metal, $M=$ di- or trivalent metal, $X=\mathrm{Si}^{4+}, \mathrm{Ge}^{4+}$, or $\mathrm{V}^{5+}$ ) are one of the main rockforming minerals in the Earth's crust [23-26] and themselves gained considerable interest due to the appearance of various interesting physical phenomena arising from their highly versatile crystal structure [27-29]. The pyroxene structure of monoclinic $\mathrm{CoGeO}_{3}$ consists of $\mathrm{Co}^{2+}$ ions that are forming $\mathrm{CoO}_{6}$ octahedral zigzag chains running in the $c$ direction

\footnotetext{
*Komarek@cpfs.mpg.de
}

Published by the American Physical Society under the terms of the Creative Commons Attribution 4.0 International license. Further distribution of this work must maintain attribution to the author(s) and the published article's title, journal citation, and DOI. Open access publication funded by the Max Planck Society. (see Ref. [30] and Fig. 1). These chains are located on an almost rectangular lattice in a plane perpendicular to the chains and there is no obvious geometric frustration in the lattice. The system orders antiferromagnetically below $T_{N} \sim$ $33.5 \mathrm{~K}$ with an Ising-like character as is indicated by $\sim 1 \mu_{B}$ enhanced effective magnetic moments (compared to the expected spin-only value) and by the very anisotropic magnetic susceptibility with Weiss temperatures of opposite signs for $H \| c$ and $H \perp c$ [30].

In this Letter, we report on our discovery of the emergence of extremely well-defined $1 / 3$ magnetization plateaus in $\mathrm{CoGeO}_{3}$ single crystals. The $1 / 3$ value is surprising since for chains on a rectangular lattice one would rather expect a value of $1 / 2$ [31]. This discovery enlarges the class of such plateau systems. Moreover, we have succeeded by using neutron scattering experiments on large single crystals to resolve the real magnetic structure in this $1 / 3 M_{S}$ phase and used this information to develop a microscopic model for the formation of these $1 / 3$ plateaus.

The floating-zone growth and characterization of monoclinic $\mathrm{CoGeO}_{3}$ is described in Ref. [30]. Magnetization measurements were performed by using the ACMS option in a physical properties measurement system (PPMS, Quantum Design) equipped with a magnet for fields up to $9 \mathrm{~T}$. The measurements of the specific heat were carried out using a standard thermal relaxation calorimetric method in a PPMS. The dielectric properties of $\mathrm{CoGeO}_{3}$ were measured on a plate $(\perp c)$ with $0.5 \mathrm{~mm}$ thickness that was coated with silver paint on both sides. Its capacitance was measured using a high-precision bridge (AH2700A, Andeen-Hagerling, Inc.). For measuring $\varepsilon(H)$ the sample was zero-field cooled to $10 \mathrm{~K}$ before starting to scan $H$ from/to $\pm 9 \mathrm{~T}$ with a rate of 30 $\mathrm{Oe} / \mathrm{s}$. Single-crystal neutron diffraction measurements have 

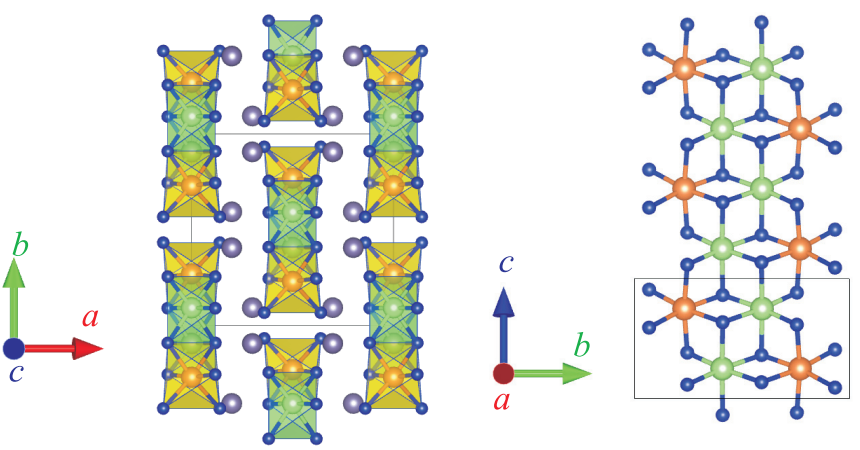

FIG. 1. Crystal structure of $\mathrm{CoGeO}_{3}$ (from the $c$ direction); a chain running along the $c$ axis is shown on the right. Green: Co1; dark yellow: $\mathrm{Co} 2$; blue: Oxygen; gray: Ge. The high-temperature polymorph of $\mathrm{CoGeO}_{3}$ has a monoclinic crystal structure with space group $C 2 / c[a=9.6623(2) \AA, b=8.9928(2) \AA, c=5.16980(10)$ $\left.\AA, \beta=101.2785(10)^{\circ}\right][30]$.
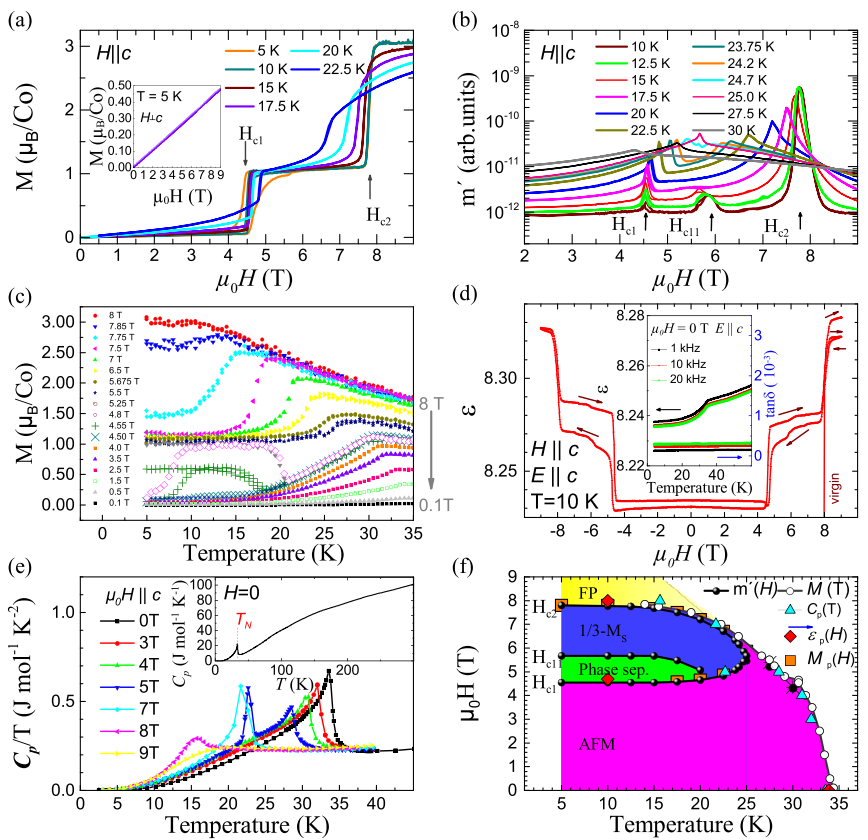

FIG. 2. Phase diagram: (a) Magnetization $M(H)$ of $\mathrm{CoGeO}_{3}$ for $H \| c$. (Data obtained by ramping up to $9 \mathrm{~T}$ and down to $0 \mathrm{~T}$ after virgin zero-field cooling from $200 \mathrm{~K}$ down.) Pronounced 1/3 magnetization plateaus can be seen. Inset: The magnetization for $H \perp c$ at $5 \mathrm{~K}$ as a function of (increasing and decreasing) magnetic field which is linear in $H$ and unsaturated up to $9 \mathrm{~T}$. (b) Real part of the ac susceptibility as a function of applied magnetic field (ac frequency: $100 \mathrm{~Hz}$; ac field: $10 \mathrm{Oe}$ ). (c) The magnetization for various fields $H \| c$. The shift of the order temperature with magnetic field can be seen. At $\sim 8 \mathrm{~T}$ the order is completely suppressed and the system enters the field-polarized (FP) regime. At intermediate fields the occurrence of the $1 / 3$ magnetization plateau becomes visible. (d) Field dependence of dielectric constant $\varepsilon$ at $T=10 \mathrm{~K}$ ( $\mu_{0} H$ was scanned in the range of -9 and $9 \mathrm{~T}$ with a rate of $30 \mathrm{Oe} / \mathrm{s})$. Inset: Temperature dependence of $\varepsilon$ and the corresponding dielectric loss $\tan \delta$ in zero field. (e) Specific heat $C_{p}(T)$ of $\mathrm{CoGeO}_{3}$ measured for different external fields $(H=0-9 \mathrm{~T})$ along the $c$ axis. (f) Magnetic phase diagram of $\mathrm{CoGeO}_{3}$ for $H \| c$. (Lines are guide to the eyes.) (a)

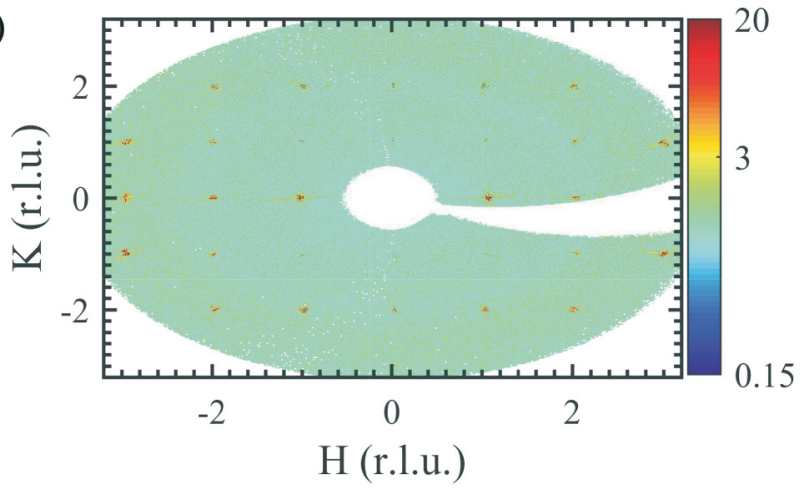

(b)

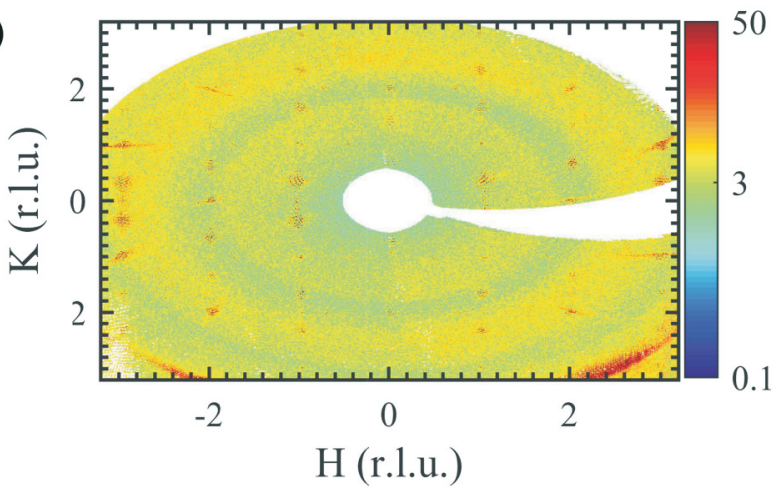

FIG. 3. Neutron scattering intensity maps within the $H K 0$ scattering plane measured at (a) $0 \mathrm{~T}$ and (b) $6.5 \mathrm{~T}$ using an area detector.

been performed on the $6 \mathrm{~T} 2$ diffractometer at Laboratoire Léon Brillouin (LLB), Saclay, France. A twinned single crystal $(\sim 5 \mathrm{~mm} \times 5 \mathrm{~mm} \times 5 \mathrm{~mm})$ was measured in fields up to $6.5 \mathrm{~T}$ $(H \| c)$. The components out of the $a^{*} b^{*}(H K)$ scattering plane could be reached by lifting of the detector. An area detector was used for the mapping of the $H K$ plane $(\lambda=$ $2.45 \AA$ ). The integrated intensities of the zero-field phase were measured by a single counter after a zero-field-cooling (ZFC) process, and after a field-cooling (FC) process for the $6.5 \mathrm{~T}$ phase $(\lambda=0.9 \AA) .72$ nuclear and 151 magnetic reflections were measured in zero field (ZF), and 196 magnetic reflections were measured at $6.5 \mathrm{~T}$. A nuclear structure refinement yields a volume fraction of 1:1.10(2) for the twin domains. The FULLPROF program package was used for magnetic structure determination.

Direction-dependent magnetization measurements on our $\mathrm{CoGeO}_{3}$ single crystals at low temperatures reveal a sharp steplike increase of the magnetization at $H_{c 1} \sim 4.5 \mathrm{~T}$ for fields parallel to the $c$ axis [see Fig. 2(a)] (for fields $H$ perpendicular to the $c$ axis a linear $M-H$ relationship can be observed as is shown in the inset). After this first jump of the magnetization $M$ a value of roughly $1 \mu_{B}$ per $\mathrm{Co}^{2+}$ ion is attained and a very well-pronounced magnetization plateau appears up to the next critical field $H_{c 2} \sim 8 \mathrm{~T}$ where the system becomes fully field polarized (FP) with $M \sim 3 \mu_{B}$ per $\mathrm{Co}^{2+}$ ion. These observations resemble the ones in $\mathrm{Ca}_{3} \mathrm{Co}_{2} \mathrm{O}_{6}$ [6-10] and $\mathrm{CoV}_{2} \mathrm{O}_{6}$ [32-38]. However, the detailed underlying magnetic structure was not directly measured in the magnetization plateau $(1 / 3$ $M_{S}$ ) phase although the zero-field antiferromagnetic (AFM) phase was determined accurately. 


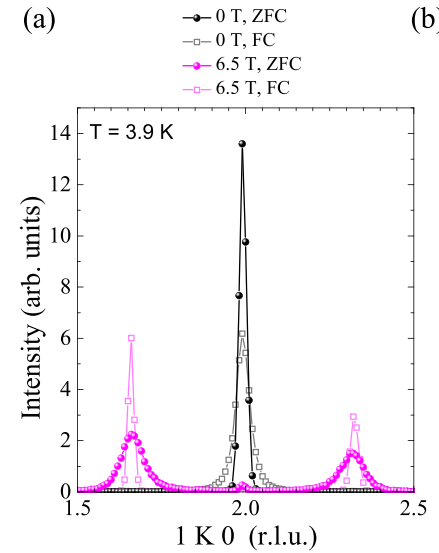

(b)

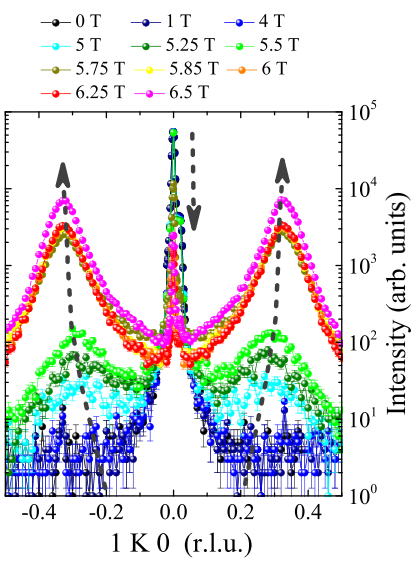

FIG. 4. (a) Neutron scans across the magnetic peak positions in the $K$ direction. (b) A detailed magnetic field dependence (for a ZFC process).

We established the entire magnetic phase diagram of $\mathrm{CoGeO}_{3}$ in great detail with dc magnetization, ac susceptibility, specific heat, and dielectric measurements [see Figs. 2(a)-2(f)]. Besides the AFM phase, the $1 / 3 M_{S}$ phase, and the FP phase, we observe yet another phase that we assign to magnetic phase separation [see Fig. 2(f)].

Furthermore, we performed neutron scattering experiments-first at $3 \mathrm{~K}$ after a zero-field-cooling (ZFC) process [see Fig. 3(a)]. In zero field (ZF) superlattice reflections with odd values of $H+K$ appear. This indicates a breaking of the $C$ centering due to the magnetic ordering. (Superlattice reflections with $|L|>0.05$ were absent). A magnetic propagation vector $\mathbf{k}_{\mathrm{ZF}}=\mathbf{k}_{1}=(100)$ is in agreement with these observations and also with powder diffraction data [22]. Then we applied an external magnetic field of $6.5 \mathrm{~T}$ along the crystallographic $c$ axis and observed a magnetic peak splitting in the $K$ direction when entering the regime of the $1 / 3 M_{S}$ phase. As a consequence the magnetic propagation vector in the $1 / 3 M_{S}$ phase changes to $\mathbf{k}_{6.5 \mathrm{~T}}=\mathbf{k}_{2}=\left(\begin{array}{lll}1 & 1 / 3 & 0\end{array}\right)$ [see Fig. 3(b)]. Scans along the $K$ direction are shown in Fig. 4(a). As can be seen in Fig. 4(a), the magnetic peaks at third-integer positions are distinctly broader after an initial ZFC process than after a field-cooling (FC) process (and vice versa for the integer peak). In Fig. 4(b) a more detailed field dependence is shown. During a ZFC process magnetic phase separation appears in $\mathrm{CoGeO}_{3}$ with two magnetic phases phases-with $\mathbf{k}_{1}$ and $\mathbf{k}_{2}$-appearing simultaneously. The peak widths of roughly $\sim 0.07$ reciprocal lattice units (r.l.u.) at $6.5 \mathrm{~T}$ indicate short-range magnetic correlations and, hence, a magnetic
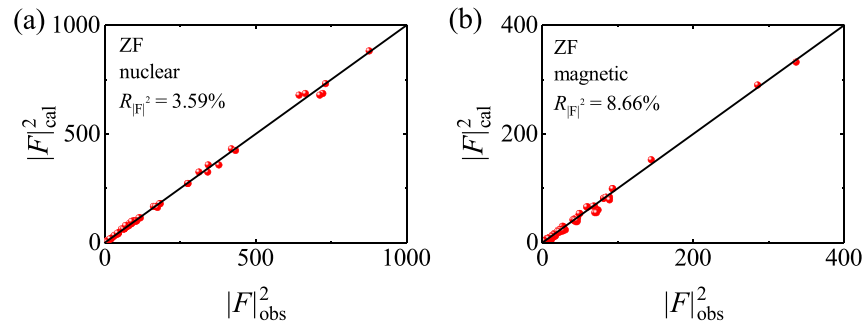

(c)

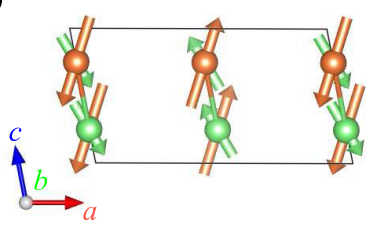

(d)

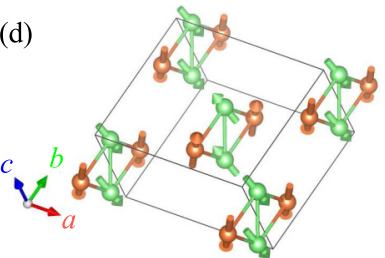

(e)
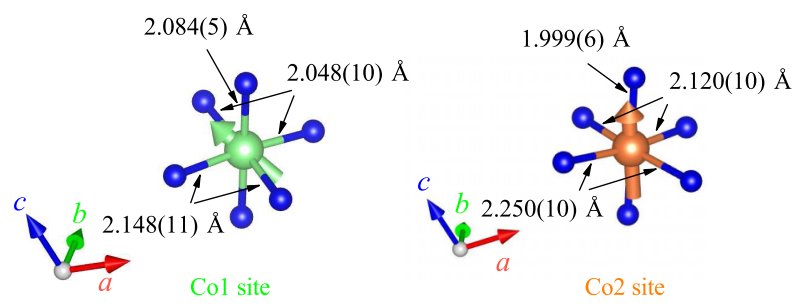

FIG. 5. Neutron refinement results for the zero-field phase: Calculated structure factor $|F|_{\text {cal }}^{2}$ against the observed structure factor $|F|_{\text {obs }}^{2}$ for (a) the nuclear structure and (b) the magnetic structure refinement at $0 \mathrm{~T}$. The corresponding magnetic structure is shown in (c) in a view from the $b$ direction, and in (d) for a different perspective. (e) Local coordination of the two Co sites; green: Co1; dark yellow: Co2; blue: oxygen.

(nano) phase separation-with the coexistence of the AFM phase and the $1 / 3 M_{S}$ phase.

Finally, we analyzed the spin structure in the ZF AFM phase and in the $1 / 3 M_{S}$ phase. For space group $C 2 / c$ and propagation vector $\mathbf{k}_{1}=(100)$, the magnetic representation for the Co ions at the $4 e$ site decomposes into four onedimensional irreducible representations (IRs) as $\Gamma=\Gamma_{1}+$ $\Gamma_{2}+2 \Gamma_{3}+2 \Gamma_{4}$ (see Table I for the corresponding basis vectors). For a second-order transition an ordering of the two Co ions with the same IR is expected. Finally, the AFM structure can be described by $\Gamma_{3}$ with moments aligned within the ac plane. Plots of $\left|F_{\text {cal }}\right|^{2}$ vs $\left|F_{\text {obs }}\right|^{2}$ are shown in Figs. 5(a) and 5(b) and the observed spin structure is shown in Figs. 5(c) and 5(d); magnetic moments are listed in Table II. The Co1 and $\mathrm{Co} 2$ ions are aligned ferromagnetically along $c$ but antiferromagnetically along $a$. The moments of the $\mathrm{Co} 2$ ions point

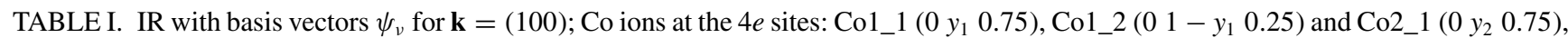
Co2_2 (0 $\left.1-y_{2} 0.25\right)$ with $y_{1}=0.0922$ and $y_{2}=0.2703$.

\begin{tabular}{|c|c|c|c|c|c|c|}
\hline IRs & $\psi_{v}$ & Coi_1 & Coi_2 & $\psi_{v}$ & Coi_1 & Coi_2 \\
\hline$\Gamma_{1}$ & $\psi_{1}$ & $\left(\begin{array}{lll}0 & 1 & 0\end{array}\right)$ & $\left(\begin{array}{lll}0 & 1 & 0\end{array}\right)$ & & & \\
\hline$\Gamma_{2}$ & $\psi_{2}$ & $\left(\begin{array}{lll}0 & 1 & 0\end{array}\right)$ & $(0-10)$ & & & \\
\hline$\Gamma_{3}$ & $\psi_{3}$ & $\left(\begin{array}{lll}1 & 0 & 0\end{array}\right)$ & $\left(\begin{array}{lll}1 & 0 & 0\end{array}\right)$ & $\psi_{4}$ & $\left(\begin{array}{lll}0 & 0 & 1\end{array}\right)$ & $\left(\begin{array}{lll}\left(\begin{array}{l}0 \\
0\end{array}\right. & 1\end{array}\right)$ \\
\hline$\Gamma_{4}$ & $\psi_{5}$ & $\left(\begin{array}{lll}1 & 0 & 0\end{array}\right)$ & $\left(\begin{array}{lll}-1 & 0 & 0\end{array}\right)$ & $\psi_{6}$ & $\left(\begin{array}{lll}0 & 0 & 1\end{array}\right)$ & $\left(\begin{array}{lll}0 & 0 & -1\end{array}\right)$ \\
\hline
\end{tabular}


TABLE II. Refinement results of the magnetic structure for $\mathrm{CoGeO}_{3}$ at zero field and at $6.5 \mathrm{~T}$; values in units of $\mu_{B}$.

\begin{tabular}{|c|c|c|c|c|c|c|c|}
\hline & & $0 \mathrm{~T}$ & $6.5 \mathrm{~T}$ & & & $0 \mathrm{~T}$ & $6.5 \mathrm{~T}$ \\
\hline \multirow[t]{3}{*}{ Co1_1 } & $M_{a}$ & $0.98(7)$ & $0.98(7)$ & \multirow[t]{3}{*}{$\mathrm{Co} 2 \_1$} & $M_{a}$ & $-2.13(7)$ & $2.09(8)$ \\
\hline & $M_{c}$ & $-2.51(3)$ & $2.49(4)$ & & $M_{c}$ & $-4.27(3)$ & $4.12(3)$ \\
\hline & $M_{\text {tot }}$ & 2.86 & 2.85 & & $M_{\mathrm{tot}}$ & 4.39 & 4.24 \\
\hline
\end{tabular}

along the shortest $\mathrm{Co}-\mathrm{O}$ bond direction. The Co1 moments attain their spin-only values whereas an additional orbital contribution [39-41] has to be considered for the Co2 moments.

The spin structure of the $1 / 3 M_{S}$ phase $(a \times 3 b \times c)$ could be determined as well (see Fig. 6). Compared to the AFM structure, the moments in entire Co chains are flipped in a way that ferromagnetically ordered chains are aligned within planes that are running in the ac direction and with inverted magnetic moments in every third of these planes with their sizes remaining basically the same as for the zero-field phase (see Table II). The total value of the net magnetization at $6.5 \mathrm{~T}$ amounts to $M_{c}=(2.49+2.49-2.49+4.12+4.12-$ 4.12) $/ 6 \mu_{B} / \mathrm{Co}=1.1 \mu_{B} / \mathrm{Co}$, which is consistent with the observation of $1 / 3$ magnetization plateaus.

Our study unravels the nature of a $1 / 3$ magnetization plateau phase which emerges from the zero-field AFM structure by flipping half of all sheets of ferromagnetic chains in order to minimize the energy at high magnetic fields. Although half of all chains are flipped, only one third of all of the antiferromagnetic alignments of nearest-neighboring Co chains of the ZF magnetic structure are lost. This is realized by the simultaneous flipping of three neighboring layers of Co chains. As one can see from the top view in Fig. 6(c)
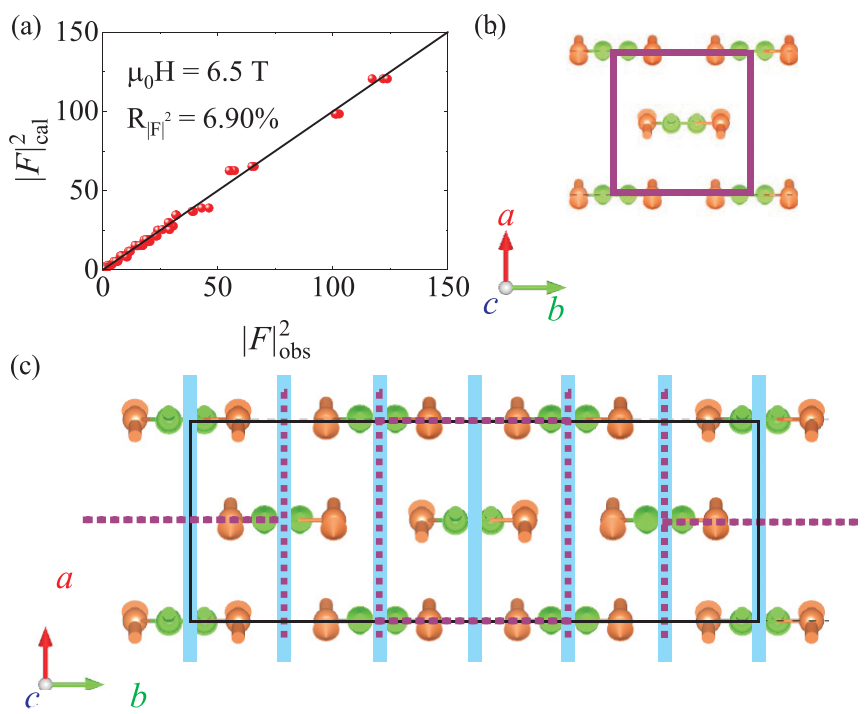

FIG. 6. Neutron refinement results for the $1 / 3$ magnetization plateau phase: (a) Calculated structure factor $|F|_{\text {cal }}^{2}$ against the observed structure factor $|F|_{\text {obs }}^{2}$ for the magnetic structure refinement at 6.5 T. (b) Zero-field magnetic structure. The purple lines indicate the AFM unit cell. (c) The magnetic structure at $6.5 \mathrm{~T}$ with an $a \times 3 b \times c$ magnetic unit cell (black lines). The light blue lines indicate planes of ferromagnetically aligned Co chains. The dashed purple lines indicate regions where the spins are aligned as in the zero-field magnetic unit cell. there exist always three neighboring layers of Co spins (light blue lines) that still show the ZF AFM ordering pattern within the $1 / 3 M_{S}$ phase. This situation resembles the creation of antiferromagnetic domains which are in one direction of the size of one unit cell. In this picture the net ferromagnetic moment appears at the "domain wall boundaries."

If one plane more or one plane less would be flipped the net moment would be zero, whereas the amount of AFM alignments would stay the same. Therefore, the $1 / 3 M_{S}$ phase can be lower in energy compared to such other magnetic phases. Moreover, it could explain why there is not yet another intermediate magnetization plateau phase. At the verge of antiferromagnetism and the $1 / 3 M_{S}$ phase, a magnetic (nano) phase separation appears which shows that other magnetic structures where more than three layers are flipped at a time are not energetically favorable compared to a magnetic phase separation with the AFM phase and 1/3 $M_{S}$ phase.

This energetic stability of the $1 / 3 M_{S}$ phase can be further supported by a theoretical model where one treats each predominantly ferromagnetic Co chain $[42,43]$ running in the $c$ direction as a single classical spin. In the $a b$ plane these spins form an effective anisotropic square (in general rhombic) lattice (see the inset in Fig. 7) with the strong nearest-neighbor (NN) exchange interaction $J_{1}$ and one weaker diagonal nextnearest-neighbor $\left(\mathrm{NNN}^{\prime}\right)$ exchange interaction $J_{2}$. The other diagonal exchange interaction $J_{3}$ shall be that weak, $J_{3} \ll$

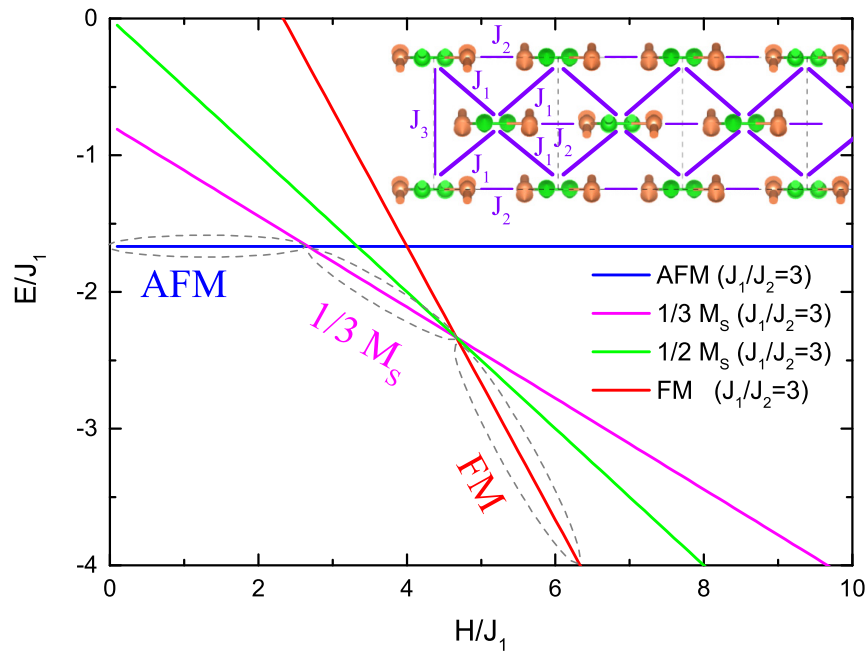

FIG. 7. Calculated energies for an anisotropic frustrated square lattice with one diagonal exchange interaction. The $1 / 2 M_{S}$ phase is never stable. For a ratio of the values of AFM NN- and $\mathrm{NNN}$-exchange interactions around 3 the expected phase diagram resembles the one shown in Fig. 2(f). The inset visualizes the underlying model of a frustrated square lattice with diagonal exchange. 
$J_{2}$, that we can neglect it. This model is justified because $\mathrm{CoGeO}_{3}$ consists of an $\mathrm{Co} 1$ zigzag chain with further $\mathrm{Co} 2$ ions attached to these chains. Thus, the distances between two chains are quite short in one diagonal direction $\| b\left(J_{2}\right)$ but much larger in the other diagonal direction $\| a\left(J_{3}\right)$ (see Fig. 7). For this model with Ising spins (as is typical for $\mathrm{Co}^{2+}$ ions), $H=J_{1} \sum_{\mathrm{NN}} S_{i} S_{j}+J_{2} \sum_{\mathrm{NNN}^{\prime}} S_{i} S_{j}-H S$, one can calculate the energies for the AFM phase, for the $1 / 3$ $M_{S}$ phase that we determined by means of neutron diffraction, for the field-polarized (FM) phase, and also for a theoretical $1 / 2 M_{S}$ phase. The energies for these different phases are plotted in Fig. 7. Obviously, a $1 / 2 M_{S}$ phase is not stable for any external field $H$. Instead, a phase diagram that is resembling our observations in Fig. 2(f) can be expected for this frustrated (anisotropic) square lattice with one diagonal exchange. Without the need for detailed $a b$ initio calculations our simple model calculations are already sufficient to clarify the origin of the $1 / 3$ magnetization plateau phase.
Concluding, we observed a very well-pronounced $1 / 3$ magnetization plateau in the synthetic pyroxene material $\mathrm{CoGeO}_{3}$. The nature of the $1 / 3 M_{S}$ plateau phase could be unraveled by means of single-crystal neutron diffraction. The minimization of energy in an external field $H \| c$ (the magnetic easy axis direction) is realized by a modulated magnetic structure with a $1 / 3$-integer magnetic propagation vector that resembles a regular formation of domain walls within the original Néel structure of the effective Ising square lattice. A model based on a frustrated anisotropic square lattice with one diagonal exchange reveals the geometrically frustrated nature of this system.

We thank P. Thalmeier for fruitful discussions. The research in Dresden was partially supported by the Deutsche Forschungsgemeinschaft through SFB 1143 (Project-ID 247310070). The work of D.I.K. was funded by the Deutsche Forschungsgemeinschaft (DFG, German Research Foundation) Project No. 277146847 - CRC 1238.
[1] M. Takigawa and F. Mila, Magnetization plateaus, in Introduction to Frustrated Magnetism: Materials, Experiments, Theory, edited by C. Lacroix, P. Mendels, and F. Mila (Springer, Berlin, 2011), pp. 241-267.

[2] B. Schmidt and P. Thalmeier, Phys. Rep. 703, 1 (2017).

[3] T. Sakakibara, T. Tayama, Z. Hiroi, K. Matsuhira, and S. Takagi, Phys. Rev. Lett. 90, 207205 (2003).

[4] H. Ueda, H. A. Katori, H. Mitamura, T. Goto, and H. Takagi, Phys. Rev. Lett. 94, 047202 (2005).

[5] Y. Shirata, H. Tanaka, A. Matsuo, and K. Kindo, Phys. Rev. Lett. 108, 057205 (2012).

[6] S. Aasland, H. Fjellvåg, and B. Hauback, Solid State Commun. 101, 187 (1997).

[7] H. Kageyama, K. Yoshimura, K. Kosuge, M. Azuma, M. Takano, H. Mitamura, and T. Goto, J. Phys. Soc. Jpn. 66, 3996 (1997).

[8] A. Maignan, C. Michel, A. C. Masset, C. Martin, and B. Raveau, Eur. Phys. J. B 15, 657 (2000).

[9] V. Hardy, M. R. Lees, O. A. Petrenko, D. McK. Paul, D. Flahaut, S. Hébert, and A. Maignan, Phys. Rev. B 70, 064424 (2004).

[10] A. Maignan, V. Hardy, S. Hébert, M. Drillon, M. R. Lees, O. Petrenko, D. McK. Paul, and D. Khomskii, J. Mater. Chem. 14, 1231 (2004).

[11] Y. B. Kudasov, Phys. Rev. Lett. 96, 027212 (2006).

[12] S. Agrestini, C. L. Fleck, L. C. Chapon, C. Mazzoli, A. Bombardi, M. R. Lees, and O. A. Petrenko, Phys. Rev. Lett. 106, 197204 (2011).

[13] S. Agrestini, L. C. Chapon, A. Daoud-Aladine, J. Schefer, A. Gukasov, C. Mazzoli, M. R. Lees, and O. A. Petrenko, Phys. Rev. Lett. 101, 097207 (2008).

[14] H. Wu, M. W. Haverkort, Z. Hu, D. I. Khomskii, and L. H. Tjeng, Phys. Rev. Lett. 95, 186401 (2005).

[15] B. Leedahl, M. Sundermann, A. Amorese, A. Severing, H. Gretarsson, L. Zhang, A. C. Komarek, A. Maignan, M. W. Haverkort, and L. H. Tjeng, Nat. Commun. 10, 5447 (2019).
[16] H. Kageyama, K. Yoshimura, R. Stern, N. V. Mushnikov, K. Onizuka, M. Kato, K. Kosuge, C. P. Slichter, T. Goto, and Y. Ueda, Phys. Rev. Lett. 82, 3168 (1999).

[17] K. Kodama, M. Takigawa, M. Horvatić, C. Berthier, H Kageyama, Y. Ueda, S. Miyahara, F. Becca, and F. Mila, Science 298, 395 (2002).

[18] Y. H. Matsuda, N. Abe, S. Takeyama, H. Kageyama, P. Corboz, A. Honecker, S. R. Manmana, G. R. Foltin, K. P. Schmidt, and F. Mila, Phys. Rev. Lett. 111, 137204 (2013).

[19] P. Corboz and F. Mila, Phys. Rev. Lett. 112, 147203 (2014).

[20] S. Yoshii, T. Yamamoto, M. Hagiwara, S. Michimura, A. Shigekawa, F. Iga, T. Takabatake, and K. Kindo, Phys. Rev. Lett. 101, 087202 (2008).

[21] S. Yoshii, K. Ohoyama, K. Kurosawa, H. Nojiri, M. Matsuda, P. Frings, F. Duc, B. Vignolle, G. L. J. A. Rikken, L.-P. Regnault, S. Michimura, and F. Iga, Phys. Rev. Lett. 103, 077203 (2009).

[22] G. J. Redhammer, A. Senyshyn, G. Tippelt, C. Pietzonka, G. Roth, and G. Amthauer, Phys. Chem. Miner. 37, 311 (2010).

[23] N. Morimoto, J. Fabries, A. K. Ferguson, I. V. Ginzburg, M. Ross, F. A. Seifert, J. Zussman, K. Aoki, and G. Gottardi, Am. Mineral. 73, 1123 (1988).

[24] D. H. Lindsley, Am. Mineral. 68, 477 (1983).

[25] F. Boyd, Geochim. Cosmochim. Acta 37, 2533 (1973).

[26] J. M. Warren and E. H. Hauri, J. Geophys. Res.: Solid Earth 119, 1851 (2014).

[27] A. N. Vasiliev, O. L. Ignatchik, A. N. Sokolov, Z. Hiroi, M. Isobe, and Y. Ueda, Phys. Rev. B 72, 012412 (2005).

[28] S. V. Streltsov, O. A. Popova, and D. I. Khomskii, Phys. Rev. Lett. 96, 249701 (2006).

[29] S. Jodlauk, P. Becker, J. A. Mydosh, D. I. Khomskii, T. Lorenz, S. V. Streltsov, D. C. Hezel, and L. Bohatý, J. Phys.: Condens. Matter 19, 432201 (2007).

[30] L. Zhao, Z. Hu, H. Guo, C. Geibel, H. J. Lin, C. T. Chen, D. I. Khomskii, L. H. Tjeng, and A. C. Komarek, Crystals 11, 378 (2021).

[31] T. Coletta, M. E. Zhitomirsky, and F. Mila, Phys. Rev. B 87, 060407(R) (2013). 
[32] Z. Z. He, J. Yamaura, Y. Ueda, and W. Cheng, J. Am. Chem. Soc. 131, 7554 (2009).

[33] S. A. J. Kimber, H. Mutka, T. Chatterji, T. Hofmann, P. F. Henry, H. N. Bordallo, D. N. Argyriou, and J. P. Attfield, Phys. Rev. B 84, 104425 (2011).

[34] M. Lenertz, J. Alaria, D. Stoeffler, S. Colis, A. Dinia, O. Mentré, G. André, F. Porcher, and E. Suard, Phys. Rev. B 86, 214428 (2012).

[35] M. Lenertz, J. Alaria, D. Stoeffler, S. Colis, and A. Dinia, J. Phys. Chem. C 115, 17190 (2011).

[36] Y. Drees, S. Agrestini, O. Zaharko, and A. Komarek, Cryst. Growth Des. 15, 1168 (2015).

[37] M. Markkula, A. M. Arevalo-Lopez, and J. P. Attfield, J. Solid State Chem. 192, 390 (2012).

[38] N. Hollmann, S. Agrestini, Z. Hu, Z. He, M. Schmidt, C.-Y. Kuo, M. Rotter, A. A. Nugroho, V. Sessi, A. Tanaka, N. B. Brookes, and L. H. Tjeng, Phys. Rev. B 89, 201101(R) (2014).

[39] S. I. Csiszar, M. W. Haverkort, Z. Hu, A. Tanaka, H. H. Hsieh, H.-J. Lin, C. T. Chen, T. Hibma, and L. H. Tjeng, Phys. Rev. Lett. 95, 187205 (2005).
[40] T. Burnus, Z. Hu, H. H. Hsieh, V. L. J. Joly, P. A. Joy, M. W. Haverkort, H. Wu, A. Tanaka, H.-J. Lin, C. T. Chen, and L. H. Tjeng, Phys. Rev. B 77, 125124 (2008).

[41] The local coordination of the $\mathrm{Co} 2$ ion can be approximated as a compressed octahedron of oxygens. Defining the local $x, y, z$ axes along the Co-O bonds and the compression axis as the $z$ axis, the high-spin $\mathrm{Co}^{2+} 3 d^{7}$ configuration will have (in addition to the five majority-spin orbitals) the minority-spin $x y$ occupied due to the lower crystal-field energy. The seventh orbital to be occupied is then given by the linear combination of the $y z$ and $z x$ which forms the $d_{1}$ state and thus carries a large orbital moment $[39,40]$. The local coordination of the Co1 ion is more complex, and apparently, the crystal field is such that the orbital degeneracy in the $t_{2 g}$ subshell is fully lifted with energy separations that are sufficiently large in comparison with the spin-orbit interaction.

[42] S. V. Streltsov and D. I. Khomskii, Phys. Rev. B 77, 064405 (2008).

[43] S. V. Streltsov and D. I. Khomskii, Phys. Usp. 60, 1121 (2017). 